\title{
Elevated Levels of Homocysteinesulfinic Acid in the Plasma of Patients with Amyotrophic Lateral Sclerosis: A Potential Source of Excitotoxicity?
}

\author{
Aven Lee $^{\mathrm{a}}$ Buddhika Jayakody Arachchige ${ }^{\mathrm{b}}$ Robert Henderson ${ }^{\mathrm{c}}$ \\ James Aylward ${ }^{d}$ Pamela Ann McCombe ${ }^{a, d}$ \\ ${ }^{a}$ Centre for Clinical Research, The University of Queensland, Brisbane, QLD, Australia; ${ }^{b}$ Mass Spectrometry Facility, \\ Centre for Clinical Research, The University of Queensland, Brisbane, QLD, Australia; 'Department of Neurology, \\ Royal Brisbane \& Women's Hospital, Brisbane, QLD, Australia; 'Wesley Medical Research, The Wesley Hospital, \\ Auchenflower, QLD, Australia
}

\section{Keywords}

Amyotrophic lateral sclerosis · Excitotoxicity · Sulfur amino acids $\cdot$ Homocysteinesulfinic acid

\begin{abstract}
Objectives: Excitotoxicity is thought to be involved in the pathogenesis of amyotrophic lateral sclerosis (ALS). One possible source of excitotoxicity is the presence of sulphur amino acids (SAAs). In the brain of subjects with ALS, there are increased levels of taurine. In the metabolism of methionine to taurine, excitatory sulphur amino acids (SAAs) are formed. These could potentially contribute to excitotoxicity in ALS. The present study has examined whether plasma levels of SAAs in 38 ALS patients differ from those of 30 healthy controls. Methods: Plasma levels of SAAs were measured by liquid chromatography mass spectrometry. Results: There were no significant changes in plasma cysteic acid, cysteine sulfinic acid, and homocysteic acid in ALS patients compared to healthy subjects. Significant elevations in plasma homocysteinesulfinic acid (HCSA) levels $(p<0.0001)$ were observed in the ALS patients (75.91 $\pm 15.38 \mathrm{nM}$ ) compared to healthy controls ( $54.06 \pm 8.503 \mathrm{nM}) ; 50 \%$ of the ALS patients had HCSA levels that were 1.5-2-folds higher than those of controls. Plasma levels of HCSA differed significantly ( $p=$
\end{abstract}

0.0440 ) between patients with bulbar onset and spinal onset (68.57 \pm 14.20 vs. $79.30 \pm 14.95$ nM, respectively). Conclusion: HCSA is elevated in the blood of subjects with ALS. Since HCSA can be transported from the blood to the CNS by active transport, has neurotransmitter properties, and can activate synaptic receptors including NMDAR and metabotropic glutamate receptor, it is possible that increases in HCSA could influence glutamatergic neurotransmission and potentially contribute to excitotoxicity in some ALS patients.

(c) 2021 The Author(s).

Published by S. Karger AG, Basel

\section{Introduction}

Amyotrophic lateral sclerosis (ALS) is a severe fatal neurodegenerative disease characterized by loss of motor neurons. There is a body of evidence implicating glutamate excitotoxicity as a contributing factor in ALS [1]. Interference with glutamate toxicity is the only therapeutic strategy that has proven to be of benefit in terms of slowing disease progression in ALS patients [2]. However, the source of the chronic glutamate toxicity in ALS is unclear. karger@karger.com www.karger.com/ndd

Karger $\frac{1}{\%}$
C 2021 The Author(s).

Published by S. Karger AG, Basel

This is an Open Access article licensed under the Creative Commons Attribution-NonCommercial-4.0 International License (CC BY-NC) (http://www.karger.com/Services/OpenAccessLicense), applicable to the online version of the article only. Usage and distribution for commercial purposes requires written permission.
Correspondence to:

Pamela Ann McCombe, pamela.mccombe@uq.edu.au 


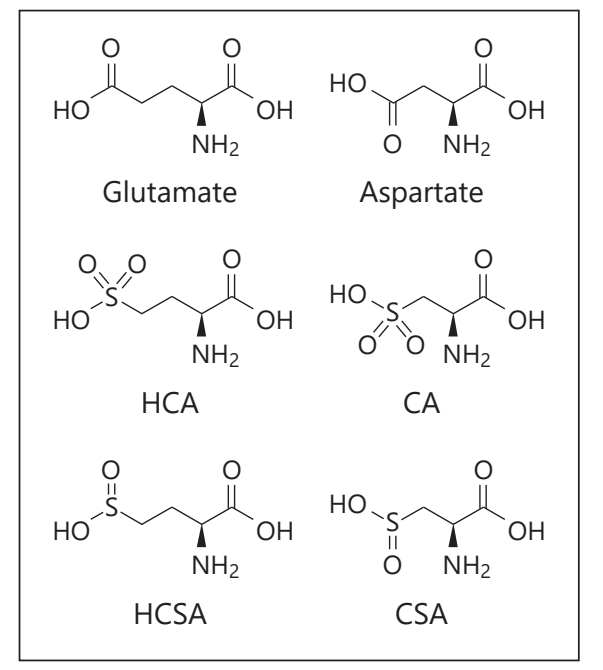

Fig. 1. Structure of glutamate, aspartate, and their sulphur-containing analogues. The carboxyl group of glutamate or aspartate is replaced by a sulphinic group for homocysteic acid (HCA) and homocysteinesulfinic acid (HCSA), or a sulphonic group for cysteic acid (CA) and cysteine sulfinic acid (CSA).

The literature also suggests that sulphur-containing amino acids (SAAs) such as cysteic acid (CA), homocysteic acid (HCA), cysteinesulfinic acid (CSA), and homocysteinesulfinic acid (HCSA) should be considered in relation to motor system-related disorders. HCA and HCSA are sulphur-containing homologues of glutamate, while CSA and CA are homologues of aspartate (Fig. 1) $[3,4]$. The pathway for CA and CSA synthesis has been identified. It involves the catabolism of dietary methionine cycled through the transmethylation pathway involving S-adenosylmethionine and S-adenosylhomocysteine to yield homocysteine, which can either be recycled to methionine or converted to cysteine. CSA and CA are then synthesized by sequential oxidation of cysteine to its corresponding sulfinic acid (CSA) and sulfonic acid (CA) using molecular oxygen. CSA and CA are precursors of taurine, and both molecules are substrates for decarboxylation by cysteine sulfinate decarboxylase [5]. ${ }^{13} \mathrm{C}-\mathrm{NMR}$ spectroscopy has revealed that the formation of taurine and hypotaurine via CSA and CA is a major pathway of cysteine metabolism in the human brain [6]. However, the biosynthetic pathway for HCA and HCSA has never been clarified but homocysteine is proposed to be the precursor of their synthesis. As no enzymes has been identified that would catalyse these reactions, the identification of these molecules in CNS tissues, therefore, has been explained by spontaneous oxidation of ho-

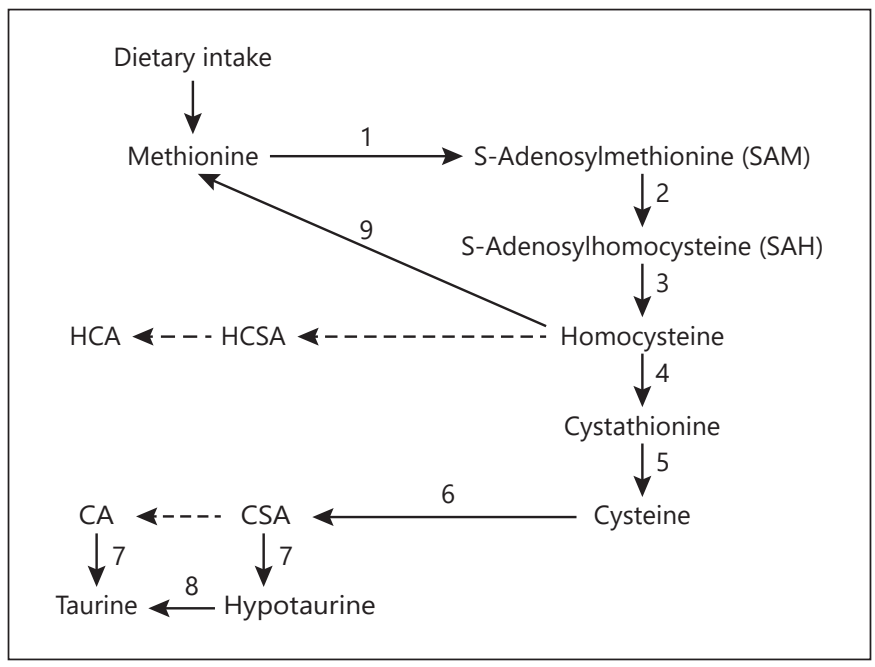

Fig. 2. Pathways of methionine metabolism and synthesis of sulphur amino acids. Black solid arrows indicate known reaction steps and dashed arrows indicate proposed steps. Numbers refer to enzymes involved in the reaction steps: 1 , methionine adenosyl transferase; 2, S-adenosylmethionine methyltransferase; 3, S-adenosylhomocysteinase; 4 , cystathionine $\beta$-synthase; 5 , cystathionine $\gamma$-lyase; 6 , cysteine dioxygenase; 7 , cysteine sulfinate decarboxylase; 8 , hypotaurine oxidase; 9 , betaine homocysteine methyltransferase and methionine synthase. Diagram adapted from Thompson and Kilpatrick [4] and Do et al. [9].

mocysteine, firstly to HCSA and then to HCA [7] (see Fig. 2).

SAAs have neurotransmitter properties and can interact with synaptic receptors such as NMDARs and metabotropic glutamate receptors to either excite or destroy neurons $[8,9]$. In the ALS motor cortex, increased levels of taurine, the final product of the metabolic pathway of SAAs, have been reported, suggesting that SAAs (the intermediates of this pathway) could also be increased in ALS [10]. Prior studies have shown that plasma homocysteine levels are significantly increased in ALS patients when compared to age- and sex-matched controls, suggesting that production of the oxidised by-product of homocysteine, HCSA, is likely to be increased among patients [11]. Thus, it is possible that these excitotoxins might play pathogenic roles in ALS. While SAAs are known to be synthesised and released from CNS tissues [4], the literature also highlights that several phyla that comprise the majority of the intestinal microbiota (Actinobacteria, Firmicutes, and Proteobacteria) are able to produce SAAs [12]. Here, we investigated whether plasma levels of SAAs in ALS patients were different from those of healthy controls. 


\section{Methods}

\section{Recruitment}

ALS patients $(n=38)$ were recruited from the Motor Neuron Disease Clinic at the Royal Brisbane and Women's Hospital. These patients had no underlying or prior disease. Healthy controls $(n=$ 30 ), with no underlying disease, who were caregivers of the ALS patients were also recruited. At entry, all ALS patients had a diagnosis of clinically definite or clinically probable ALS according to the revised El Escorial criteria. By the end of the study, 31 patients had clinically definite ALS (21 patients had died of ALS and 10 were alive with clinically definite ALS). Of the remainder, 5 were alive with upper motor neurone predominant disease, 1 had died of lower motor neurone predominant disease and 1 was alive with flail limb phenotype. We recorded the age, the site of onset and disease duration from the time of onset of symptoms to the date of blood collection. The burden of disease at the time of blood collection was measured as the ALSFRS-R. We noted whether disease was sporadic or familial and the genotype if this was known but there was no systemic genetic screening for ALS causative genes.

\section{Plasma Samples}

Samples of whole blood (fasting) were collected in BD EDTA coated vacutainers, and plasma was immediately separated by centrifugation at $1,500 \mathrm{~g}$ for $10 \mathrm{~min}$ at $4^{\circ} \mathrm{C}$, transferred to microcentrifuge tubes, and stored at $-80^{\circ} \mathrm{C}$ until use. Proteins were precipitated by addition of $100 \mu \mathrm{L}$ of $80 \%$ acetonitrile/ $20 \%$ methanol to $55 \mu \mathrm{L}$ plasma. The mixed solution was vortexed for $2 \mathrm{~min}$ and centrifuged at $17,000 \mathrm{~g}$ for $10 \mathrm{~min}$. The supernatant was collected, dried down using a rotary speed-vac, and the pellet resuspended in $110 \mu \mathrm{L}$ of $0.1 \%$ formic acid; an aliquot of $5 \mu \mathrm{L}$ was injected into the liquid chromatography mass spectrometry (LC-MS) system for analysis.

\section{Measurements of L-CA, L-CSA, and L-HCSA}

Measurements of CA, CSA, and HCSA in plasma samples of ALS patients and healthy controls were performed by liquid chromatography multiple reaction monitoring mass spectrometry (LC-MRM-MS). The calibration curves were linear over the range examined for the respective molecules (CA: 3.0-1,500 nM, $R^{2}>$ 0.9991; CSA: $1.20-1,250 \mathrm{nM}, R^{2}>0.9995$; HCSA: $3.0-1,500 \mathrm{nM}, R^{2}$ $>0.9996)$. The assay was validated using quality control samples; the accuracy of the values determined from each quality control samples were within $100 \pm 10 \%$ of their expected values.

CA was separated on a Shimadzu Nexera UHPLC system with XB Amide C18 column $(4.6 \times 150 \mathrm{~mm})$ from Waters. The mobile phase used for separation was buffer $\mathrm{A}(0.1 \%$ formic acid in water) and buffer B (0.1\% formic acid in $100 \%$ acetonitrile). Samples (5 $\mu \mathrm{L}$ ) were loaded at $0.45 \mathrm{~mL} / \mathrm{min}$, and the separation was carried out at $50 \% \mathrm{~B}$ for $6 \mathrm{~min}$. The column oven and auto-sampler were operated at $25^{\circ} \mathrm{C}$ and $5^{\circ} \mathrm{C}$, respectively. Samples eluting from the column were monitored in positive ion mode with MRM on a Shimadzu LCMS8050 mass spectrometer equipped with electrospray ion source. The following ion source parameters were used: nebulizer gas flow $(3 \mathrm{~L} / \mathrm{min})$, heating gas flow $(10 \mathrm{~L} / \mathrm{min})$, drying gas flow $(10 \mathrm{~L} / \mathrm{min})$, heater interface temp $\left(300^{\circ} \mathrm{C}\right), \mathrm{DL}$ temp $\left(250^{\circ} \mathrm{C}\right)$, and heater block temperature $\left(400^{\circ} \mathrm{C}\right)$. Quadrupole 1 (Q1) and quadrupole $3(\mathrm{Q} 3)$ were operated at unit mass resolution at all time during the experiment. A parent mass of $168.00 \mathrm{Da}$ (Q1) was selected and fragmented at $-15.0 \mathrm{~V}$ collision energy $(\mathrm{CE})$, and a frag- ment mass of $80.90 \mathrm{Da}(\mathrm{Q} 3)$ was selected for quantification with 100 ms dwell time. Data were processed using Shimadzu LabSolutions (Quant Browser) software 5.93.

LC-MRM-MS analysis of HCSA in human plasma was the same as described above, with modifications to the HPLC and MRM settings. The injection volume and flow rate were $1 \mu \mathrm{L}$ and $0.30 \mathrm{~mL} / \mathrm{min}$, respectively. The HPLC gradient was as follows: buffer B from 5 to $50 \%$ over $0.0-5.0 \mathrm{~min}, 50 \%$ B to $95 \%$ B over $5.0-5.50$ min, column washed at $95 \%$ B from 5.50 to $7.50 \mathrm{~min}$, followed by equilibration for $2 \mathrm{~min}$ at $5 \% \mathrm{~B}$. HCSA was monitored using the precursor-product ion transitions: $168.0>121.8$ with $100 \mathrm{~ms}$ dwell time.

CSA was separated on a Shimadzu Nexera UHPLC system with a HILIC column $(4.6 \times 150 \mathrm{~mm})$ from Phenomenex. The mobile phase used for separation was buffer $\mathrm{A}(0.1 \%$ formic acid in water $)$ and buffer B ( $0.1 \%$ formic acid in $100 \%$ acetonitrile). Samples ( 10 $\mu \mathrm{L}$ ) were loaded at $0.40 \mathrm{~mL} / \mathrm{min}$, and the separation was carried out as follows: buffer B from 50 to $20 \%$ over $0.0-4.0 \mathrm{~min}$, column washed at $20 \%$ B from 4.0 to $6.0 \mathrm{~min}$, followed by equilibration for $2 \mathrm{~min}$ at $50 \% \mathrm{~B}$. The column oven and auto-sampler were operated at $25^{\circ} \mathrm{C}$ and $5^{\circ} \mathrm{C}$, respectively. Samples eluted from the column were monitored in negative ion mode with MRM on a QTRAP 5500-1 mass spectrometer (AB Sciex Instruments). The following ion source conditions were used: curtain gas flow (40), GS1 gas flow (35), GS2 gas flow (30), ion source temp $\left(400^{\circ} \mathrm{C}\right)$, ion source voltage $(-4,500 \mathrm{v})$, and De-clustering potential $(-80 \mathrm{v})$. CSA was monitored using the precursor-product ion transitions: $152.1>$ 88.1 with $5.4 \mathrm{~min}$ retention time.

\section{Measurement of L-HCA}

Plasma levels of HCA were measured using an Abbkine Human Homocysteic Acid ELISA kit, following precisely the manufacturer's instruction. This kit employs a 2-site sandwich ELISA to quantify HCA in biological fluids such as plasma and serum. The capture antibody is monoclonal, and the detection antibody is polyclonal. The calibration curve was linear over the range examined (0.5-8.0 $\left.\mathrm{nmol} / \mathrm{mL} ; R^{2}>0.998\right)$. The lower limit of quantification (LLOQ) was $0.5 \mathrm{nmol} / \mathrm{mL}(92 \mathrm{pg} / \mathrm{mL})$, and all measured blood levels of HCA in healthy controls and ALS patients were above the LLOQ.

\section{Statistics}

Plasma levels of SAAs in patients and controls were compared using the Mann-Whitney U test. Spearman's correlation test was used to assess the correlation between levels of HCSA and other variables. Power calculations were performed to guide sample size decisions; our analysis determined that a sample size of 26 or more was needed to detect moderate to strong difference effects between the 2 groups [based on an effect size $d=0.8$, $a$ err prob $=0.05$ and Power $(1-\beta$ err prob $)=0.80]$.

\section{Results}

\section{Subjects}

The demographics of our participant cohort and clinical features of patients are summarised in Table 1. The groups were well-matched with no significant differences between controls and patients. 
Table 1. Clinical details of participants in the plasma SAA study

\begin{tabular}{llll}
\hline & Healthy controls & ALS & $p$ value \\
\hline Number of participants & 30 & 38 & $\mathrm{n} / \mathrm{a}$ \\
Male: female & $18: 12(60 \%$ male $)$ & $22: 16(58 \%$ male $)$ & 0.444 \\
Age (mean \pm SD) & $58.1 \pm 8.2$ & $62.4 \pm 8.5$ & 0.062 \\
ALSFRS-R (mean \pm SD) & n/a & $36.0 \pm 5.4$ & n/a \\
Disease duration from onset (median, range), days & n/a & $889(762,1,761)$ & n/a \\
Site of onset (bulbar/upper limb/lower limb) & n/a & $12 / 8 / 18$ & n/a \\
Patients with known family history of ALS, $n$ & n/a & 4 & n/a \\
Patients with cognitive impairment, $n$ & n/a & Not determined & n/a \\
\hline
\end{tabular}

SAA, sulphur amino acid; ALS, amyotrophic lateral sclerosis.

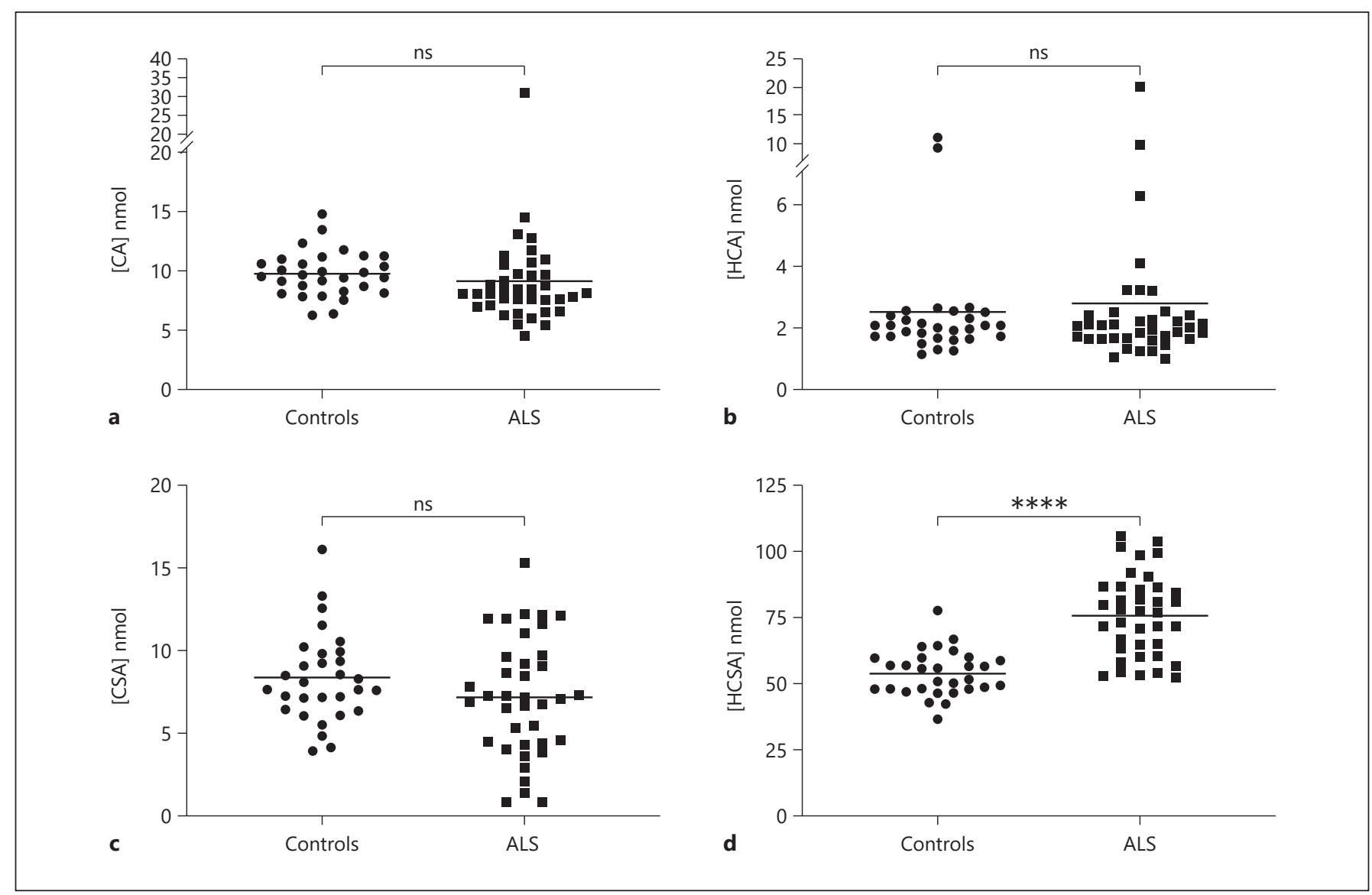

Fig. 3. Individual value plots with mean (horizontal line) to show concentrations of CA (a), HCA (b), CSA (c), and HCSA (d) in plasma from ALS patients (38) and controls $(30) ;{ }^{* * *} p<0.0001$. ALS, amyotrophic lateral sclerosis; CA, cysteic acid; HCA, homocysteic acid; CSA, cysteinesulfinic acid; HCSA, homocysteinesulfinic acid.

Concentrations of SAAs in ALS Patient Plasma

Levels of CA, CSA, and HCA in ALS patient plasma were not significantly different from those of healthy control subjects (CA: $9.137 \pm 4.252$ vs. $9.570 \pm 2.012 \mathrm{nM}, p=$
0.0550; CSA: $7.160 \pm 3.589$ vs. $8.355 \pm 2.703 \mathrm{nM}, p=$ 0.1433 ; HCA: $2.817 \pm 3.252$ vs. $2.523 \pm 2.133 \mathrm{nM}, p=$ 0.8999, respectively) (Fig. 3a-c). Plasma HCSA levels were significantly higher $(p<0.0001)$ in the ALS patient 


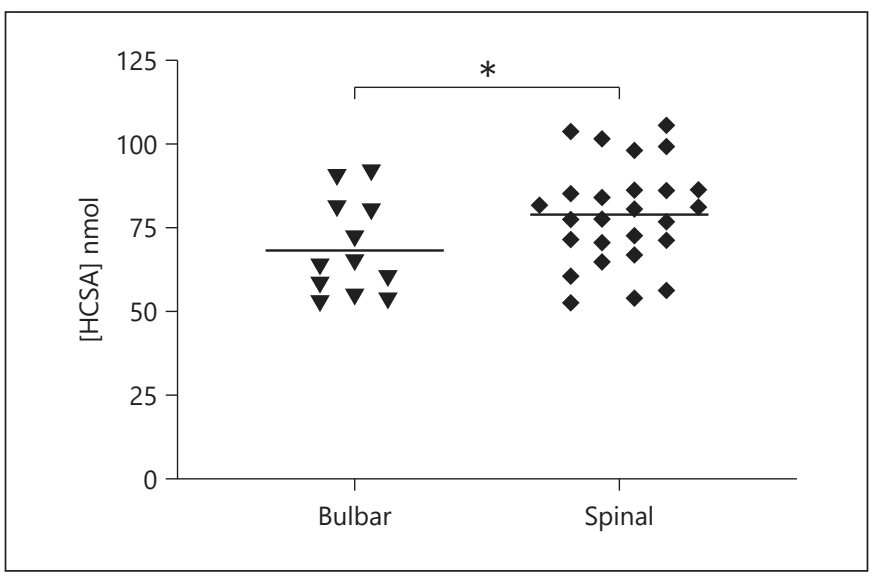

Fig. 4. Comparison of HCSA levels in plasma from patients with bulbar and spinal onset; ${ }^{*} p<0.05$. HCSA, homocysteinesulfinic acid.

group than the healthy control group $(75.91 \pm 15.38$ vs. $54.06 \pm 8.503 \mathrm{nM}$, respectively) (Fig. $3 \mathrm{~d}$ ). We found that $50 \%$ of the patients had plasma HCSA levels that were 1.5 - to 2-fold higher than those of healthy controls. Significant difference $(p=0.0440)$ between patients with bulbar onset and spinal onset was also observed in plasma levels of HCSA $(68.57 \pm 14.20$ vs. $79.30 \pm 14.95 \mathrm{nM}$, respectively) (Fig. 4). There were no significant correlation of HCSA levels with age $(r=-0.08420, p=0.6153)$, ALSFRS-R $(r=-0.1610, p=0.3342)$ or with duration of disease $(r=0.1665, p=0.3179)$.

\section{Discussion}

This study compared the levels of SAAs, which are possible sources of excitotoxicity, in the blood of patients with ALS and in controls. We measured CA, CSA, and HCSA with LC-MRM-MS and HCA with ELISA. The principal finding of this study was that HCSA is significantly elevated in the blood of many patients with ALS, compared to controls. Specifically, we showed that half of the patient cohort had plasma HCSA levels that were 1.5to 2-fold higher than those of controls. For the other SAAs, there were no differences between ALS patients and controls.

For HCSA, we also found differences between patients with bulbar and spinal onset ALS. Generally, patients with onset in different regions, but spread to other regions, fulfil the criteria for diagnosis of ALS and are considered to have the same disease. However, ALS patients are heterogeneous and have different clinical features and course of disease. It is possible that there are subgroups of patients with differing aetiology and pathogenesis of disease. Patients with bulbar and spinal onset ALS differ in age of onset and sex ratio [13] and in the incidence of cognitive impairment [14]. Our finding of different levels of HCSA could support the possibility that these subgroups have differing pathological processes.

The source of the elevated HCSA could be endogenous production. HCSA, a sulphur-containing homologue of glutamate, is an oxidised metabolite of homocysteine in humans. However, the precise mechanism involved in the biosynthesis of HCSA (and HCA) has never been clarified. Previous studies found no evidence of whether the 2 molecules are products of oxidative metabolism of homocysteine in analogy to the route of CSA and CA formation by oxidative metabolism of cysteine [15]. In the present study, we found that plasma HCSA was significantly elevated in half of the ALS patient cohort. It is worth noting that the increase in plasma HCSA was not associated with parallel increases in HCA. Since HCSA and HCA are both oxidised products of homocysteine, one would expect that levels of these 2 molecules are likely to change in tandem, especially in pathological states. It is difficult at present to speculate upon the reason for the increased HCSA but not of HCA in ALS. It may be that the increased HCSA arise by increased oxidation of homocysteine, which is known to be elevated in patients with ALS [16]; it may be that the activity of oxidases that subsequently convert HCSA to HCA is decreased in ALS. In studies where brain extracts were incubated with $\left[{ }^{35} \mathrm{~S}\right]$ homocysteine and its derivatives examined by pre-column $o$-phthalaldehyde derivatisation followed by HPLC, only a slow nonenzymic increase in HCSA was detected, whereas the level of HCA was below the limit of sensitivity [15]. Such findings suggest that the biosynthesis of HCSA and HCA from homocysteine is somewhat varied. Recent metabolomics studies also show that the two molecules do not always change in tandem in some pathological states. One study performed high-resolution metabolomics to identify changes in serum metabolites in acute myocardial infarction and found that only HCSA (and CA) levels were increased and not of HCA [17]. Another metabolomics study found increased levels of HCSA only (and not of the other SAAs) in serum samples of stroke risk patients, as compared with control subjects [18]. Our finding of HCSA as the only SAA elevated in patient plasma further suggests that at least one of the three routes for elimination of homocysteine may be perturbed. As shown in Figure 1, there are three routes for 
elimination - homocysteine can either be recycled back to methionine or converted to cystathionine for production of cysteine, which is subsequently oxidised to CA and CSA (cystathionine pathway); alternatively, homocysteine can undergoes oxidation to produce HCSA and HCA (homocysteine oxidation pathway). Our findings suggest that the homocysteine oxidation pathway is induced in ALS, resulting in more excitatory amino acids, HCSA being generated.

Much immunocytochemical studies suggest that SAAs, including CA, CSA, HCA, and HCSA are largely synthesised and released from CNS tissues [8]. Another possible source of HCSA is microbial metabolism of cysteine, which results in the production of SAAs. Eubacteria express bona fide enzymes such as cysteine dioxygenase that can oxidize cysteine to CSA; phylogenetic analysis also indicate that cysteine dioxygenase is distributed among several phyla that comprise the majority of the intestinal microbiota, including Actinobacteria, Firmicutes, and Proteobacteria [12]. Collectively, these findings suggest that a large subset of the gut microbiota could contribute to the production of CSA and possibly other SAAs. The SAAs synthesised by intestinal microbes can enter the blood via effective transport systems in the intestinal tract. In fact, CA, CSA, HCA, and HCSA are all substrates for the EAAT family of glutamate transporters [8] and EAAT3/EAAC1 is known to be expressed in the intestine [19]. However, we acknowledge that one limitation of our study is that we did not measure SAAs in the cerebrospinal fluid.

One limitation of this study is that we used LC-MRMMS for CA, CSA, and HCSA, and ELISA for HCA. However, we compared the levels of the different SAAs between patients and controls, who were measured with the same methods. We did not attempt to compare the levels of the different SAAs.

The finding of elevated HCSA levels could have implications for ALS pathogenesis since HCSA is cytotoxic when tested on cerebral cortical neurons in vitro [20]. Studies have shown that SAAs are all effective agonists at several metabotropic glutamate receptors (mGluR). HCSA, HCA, CSA, and CA are all reported to be more potent mGluR agonists than glutamate [21]. Of the four SAAs, HCSA is the most potent agonist at mGluR1, mGluR2, mGluR4, mGluR5, mGluR6, and mGluR8 [22]. Electrophysiological studies in hippocampal neurons also demonstrate HCSA can evoke activation of NMDA receptors [23]. Hyperactivation of glutamate receptors by SAAs can cause neuronal death through excitotoxicity. Examples include SAA-induced epileptic activity follow- ing direct administration to rodents [24] and SAA-induced toxicity in neuronal cell lines and spinal cord neurons [25]. Predictably, blockade of glutamate receptors was shown to prevent SAA-induced excitotoxicity [19, 26]. Thus, it is conceivable that the elevated HCSA that we have observed could act at both NMDA and nonNMDA glutamate receptors and cause excitotoxicity in some patients with ALS.

\section{Acknowledgements}

We acknowledge the support of the subjects who contributed to this study and to our research nurses Susan Heggie and Kathyrn Ann Thorpe.

\section{Statement of Ethics}

This study was approved by the Human Research Ethics Committee of the Royal Brisbane and Women's Hospital (2006/047). All patients gave written informed consent to participation.

\section{Conflict of Interest Statement}

The authors have nothing to declare.

\section{Funding Sources}

This study was supported by a Wesley Medical Research Grant awarded to P.A. McCombe and R.D. Henderson and by a grant from MND Research Australia to P.A. McCombe. There were no award numbers.

\section{Author Contributions}

Dr. Aven Lee conceived the study, carried out the experiments, and wrote the first draft of the paper. Mr. Buddhika Jayakody Arachchige carried out the experiments and contributed to writing the paper. Dr. Robert Henderson helped to recruit patients and contributed to writing the paper. Dr. James Aylward helped to conceive the study and contributed to writing the paper. Prof Pamela Ann McCombe conceived the study, helped to recruit patients, and contributed to writing the paper.

\section{Data Availability Statement}

Data are available from the authors on reasonable request. 


\section{References}

1 Le Gall L, Anakor E, Connolly O, Vijayakumar UG, Duddy WJ, Duguez S. Molecular and cellular mechanisms affected in ALS. J Pers Med. 2020;10(3):101.

2 Chiò A, Mazzini L, Mora G. Disease-modifying therapies in amyotrophic lateral sclerosis. Neuropharmacology. 2020;167:107986.

3 Olney J. Excitotoxic amino acids. Physiology. 1986;1(1):19-23.

4 Thompson GA, Kilpatrick IC. The neurotransmitter candidature of sulphur-containing excitatory amino acids in the mammalian central nervous system. Pharmacol Ther. 1996;72(1):25-36.

5 Griffith OW. Mammalian sulfur amino acid metabolism: an overview. Methods Enzymol. 1987;143:366-76.

6 Brand A, Leibfritz D, Hamprecht B, Dringen R. Metabolism of cysteine in astroglial cells: synthesis of hypotaurine and taurine. J Neurochem. 1998;71(2):827-32.

7 Kilpatrick IC, Walter SJ, Harrison IS, Evans RH, Holman RB. Rapid, automated HPLC analyese of sulphur-containing and some other neurotoxic amine acids. In: Cross AJMH, editor. High performance liquid chromatography in neuroscience resarc, IBRO methods handbook. Chichester: Lohn Wiley and Sons; 1993. p. 139-62.

8 McBean GJ. Sulfur-containing amino acids. In: Lajtha AOSS, Schousboe A, Saransaari P, editors. Handbook of neurochemistry and molecular neurobiology. Boston: Springer; 2007.

9 Do KQ, Herrling PL, Streit P, Cuénod M. Release of neuroactive substances: homocysteic acid as an endogenous agonist of the NMDA receptor. J Neural Transm. 1988;72(3):18590.

10 Yoshino Y, Koike H, Akai K. Free amino acids in motor cortex of amyotrophic lateral sclerosis. Experientia. 1979;35(2):219-20.
11 Wang HL, Fan DS. [The relation between plasma homocysteine level and amyotrophic lateral sclerosis]. Zhonghua nei ke za zhi. 2012;51(4):308-10.

12 Dominy JE Jr, Simmons CR, Karplus PA, Gehring AM, Stipanuk MH. Identification and characterization of bacterial cysteine dioxygenases: a new route of cysteine degradation for eubacteria. J Bacteriol. 2006;188(15): 5561-9.

13 McCombe PA, Henderson RD. Effects of gender in amyotrophic lateral sclerosis. Gend Med. 2010;7(6):557-70.

14 Yang T, Hou Y, Li C, Cao B, Cheng Y, Wei Q, et al. Risk factors for cognitive impairment in amyotrophic lateral sclerosis: a systematic review and meta-analysis. J Neurol Neurosurg Psychiatry. 2021;92(7):688-93.

15 Cuénod M, Do KQ, Grandes P, Morino P, Streit $\mathrm{P}$. Localization and release of homocysteic acid, an excitatory sulfur-containing amino acid. J Histochem Cytochem. 1990;38(12): 1713-5.

16 Zoccolella S, Simone IL, Lamberti P, Samarelli V, Tortelli R, Serlenga L, et al. Elevated plasma homocysteine levels in patients with amyotrophic lateral sclerosis. Neurology. 2008; 70(3):222-5.

17 Khan A, Choi Y, Back JH, Lee S, Jee SH, Park YH. High-resolution metabolomics study revealing 1-homocysteine sulfinic acid, cysteic acid, and carnitine as novel biomarkers for high acute myocardial infarction risk. Metabolism. 2020;104:154051.

18 Lee Y, Khan A, Hong S, Jee SH, Park YH. A metabolomic study on high-risk stroke patients determines low levels of serum lysine metabolites: a retrospective cohort study. Mol Biosyst. 2017;13(6):1109-20.
19 Schurr A, West CA, Heine MF, Rigor BM The neurotoxicity of sulfur-containing amino acids in energy-deprived rat hippocampal slices. Brain Res. 1993;601(1-2):317-20.

20 Frandsen A, Schousboe A, Griffiths R. Cytotoxic actions and effects on intracellular $\mathrm{Ca} 2+$ and cGMP concentrations of sulphur-containing excitatory amino acids in cultured cerebral cortical neurons. J Neurosci Res. 1993; 34(3):331-9.

21 Porter RH, Roberts PJ. Glutamate metabotropic receptor activation in neonatal rat cerebral cortex by sulphur-containing excitatory amino acids. Neurosci Lett. 1993;154(1-2): 78-80.

22 Shi Q, Savage JE, Hufeisen SJ, Rauser L, Grajkowska E, Ernsberger P, et al. L-homocysteine sulfinic acid and other acidic homocysteine derivatives are potent and selective metabotropic glutamate receptor agonists. J Pharmacol Exp Ther. 2003;305(1):131-42.

23 Patneau DK, Mayer ML. Structure-activity relationships for amino acid transmitter candidates acting at $\mathrm{N}$-methyl-D-aspartate and quisqualate receptors. J Neurosci. 1990;10(7): 2385-99.

24 Butcher SP, Cameron D, Kendall L, Griffiths R. Homocysteine-induced alterations in extracellular amino acids in rat hippocampus. Neurochem Int. 1992;20(1):75-80.

25 Mewett KN, Oakes DJ, Olverman HJ, Smith DA, Watkins JC. Pharmacology of the excitatory actions of sulphonic and sulphinic amino acids. Adv Biochem Psychopharmacol. 1983;37:163-74.

26 Jara-Prado A, Ortega-Vazquez A, MartinezRuano L, Rios C, Santamaria A. Homocysteine-induced brain lipid peroxidation: effects of NMDA receptor blockade, antioxidant treatment, and nitric oxide synthase inhibition. Neurotox Res. 2003;5(4):237-43. 